\title{
AVANCES EN EL ESTUDIO HISTÓRICO DE LA MINA DE GALENA DE Bunyola (ISLA De MallorCa)
}

En este trabajo se presentan los resultados de los análisis químicos e isotópicos realizados para determinar la signatura isotópica de la zona minera de Bunyola (isla de Mallorca), uno de los yacimientos de galena que hay en el archipiélago balear. Estos trabajos se realizaron para contextualizar los restos mineros de la zona, así como algunos conjuntos de materiales arqueológicos de plomo, cuya materia prima se atribuía a este yacimiento minero. Sin embargo, gran parte de los restos hallados sobre el terreno corresponden a la actividad minera de finales del s. XIX y principios del XX. La posibilidad de que se haya explotado el yacimiento en época antigua deberá quedar a la espera de obtener más información a través de resultados arqueológicos y analíticos.

Palabras clave: arqueominería, estudios analíticos, islas Baleares.

\section{Approach to the historical Study of The galena mine of BUnyola (MajorCa, Balearic Islands)}

This paper is to present the analytical results (chemical and isotopic) conducted to determine the fingerprint of the mining area of Bunyola (Majorca), one of the galena deposits having the Balearic Islands. These investigations were conducted to contextualize both the remnants of the mining area as to contextualize some sets of archaeological material of lead, whose raw material was attributed to this mine site. However, much of the remains in the terrain belong to the mining of the late nineteenth century and early twentieth century. Considerations about possible exploitation in ancient times must remain open until it can be determined with more archaeological and/or analytical data.

Key words: ancient mining, isotopic analysis, Balearic Islands.

El yacimiento minero de Bunyola es uno de los yacimientos de galena ya conocidos de las islas Baleares ( $c f$. Jordà 2002). Es de menor dimensión que el de s'Argentera en la isla de Eivissa, dónde desde hace unos años se llevan a cabo investigaciones para verificar una posible explotación romana del yacimiento, sin excluir la posibilidad de hallar huellas de una explotación prerromana (Ramon et al. 2011: 58). Los trabajos se desarrollan directamente sobre el terreno, además de recoger numerosas muestras para su análisis, no sólo del yacimiento de s'Argentera, sino también objetos de otros contextos arqueológicos de la isla (Jordà et al. 2011; Hermanns
2013). También se están intentando localizar otras actividades mineras mencionadas en la documentación que se conserva en el archivo del Reino de Mallorca (Palma de Mallorca), como es el caso de la zona de Es Figueral y de las minas de Sant Joan de Labritja. Uno de los principales objetivos es el de verificar la signatura isotópica del lugar y compararla con las demás conocidas, para así poder determinar la procedencia de los objetos arqueológicos de plomo y definir la dinámica y dimensión de la explotación ibicenca. Es en este contexto donde se enmarca nuestro interés por estudiar y determinar analíticamente las características del yacimiento de Bunyola. 


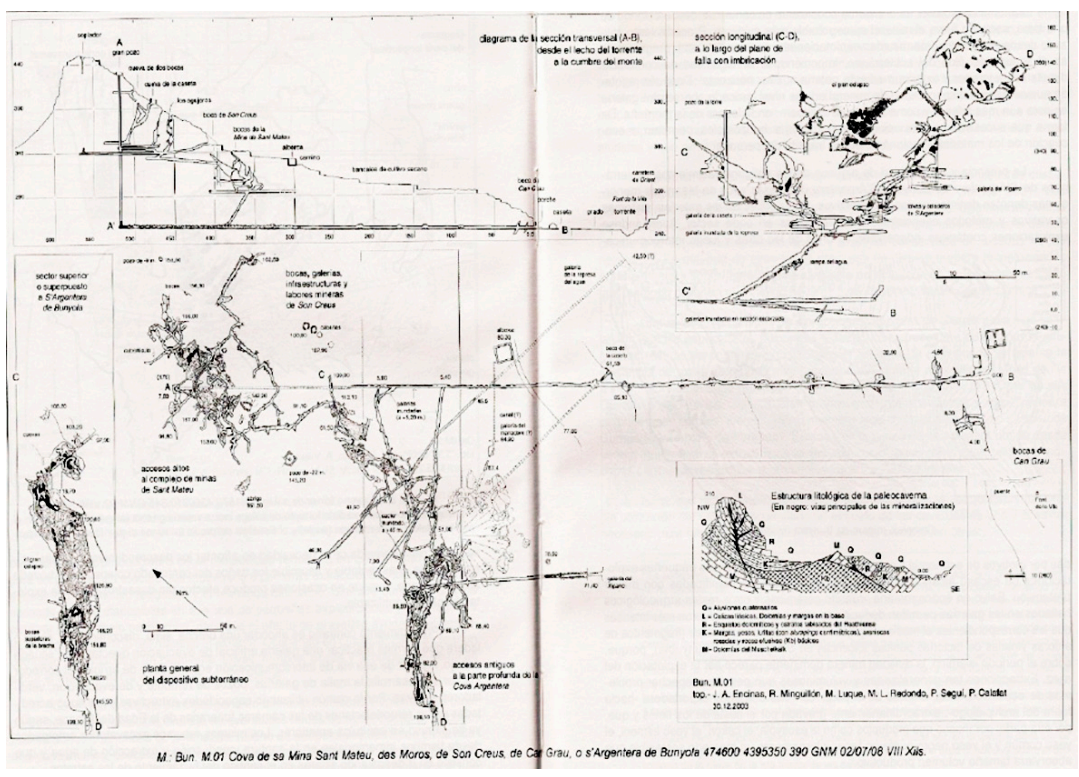

Fig. 1. Plano de las mina de San Mateo, Son Creus en Bunyola (isla de Mallorca) (Merino 1992).

En 1867, en la Estadística Minera (1867: 53) se cita el descubrimiento de un yacimiento de calamina en un banco de dolomía situado en el término de Bunyola, isla de Mallorca (Baleares). El afloramiento estaba al descubierto, en una zona hundida debido a antiguas e irregulares labores. Sin embargo, estos trabajos seguían las vetas y bolsadas de galena, no las de calamina. Se emplearon entonces algunos hombres en la renovada búsqueda y en el lavado del material plomizo que rellenaba y taponaba estas galerías. En 1868 el retroceso de la explotación minera de las islas Baleares en cuanto a la producción de lignito y calamina viene con exceso compensada con la mayor extracción de mineral de plomo, el cual dos años atrás ni siquiera figuraba en las relaciones estadísticas (Estadística Minera 1868: 46). Esta primera etapa de explotación minera moderna en Bunyola dura hasta 1870 , abandonándose en ese año unos trabajos tan desordenados como debieron ser aquellos con los se encontraron al comienzo de esta nueva etapa. La mayor parte de la obtención del plomo en las islas Baleares se centraba, por entonces, en la explotación de la zona minera de s'Argentera, en Ibiza: primero (a partir del 1870) aprovechando los escoriales antiguos, y luego procediendo a una explotación subterránea ( $c f$. Estadística Minera 1869: 42; Jordà et al. 2011).

El s. XIX, concretamente el periodo comprendido entre 1850 y 1870 , es la época dorada de la arqueología minera en la península Ibérica. En esas décadas tiene lugar un resurgimiento de la minería en la península y en las Baleares, aquí principalmente en Ibiza, y es durante las primeras décadas de la reactivación minera cuando se empiezan a producir interesantes hallazgos arqueológicos en este ámbito. Conviene estudiar los informes mineros de aquella época para rescatar descripciones de trabajos antiguos cuyos vestigios se han perdido como consecuencia de actividades posteriores. Sin embargo debemos señalar que habitualmente en aquella época, a todo trabajo antiguo se le atribuía un origen romano, tratando de realzar el valor e importancia del yacimiento minero para alentar su explotación en ese momento (de Jáuregui 1948: 89). Por tanto, esas afirmaciones deben de tomarse como un punto de partida, debiendo ser corroboradas con hallazgos arqueólogicos datables. A falta de dichos hallazgos arqueológicos (herramientas, cerámica, monedas, material orgánico), muchas veces son las diferentes huellas de explotación las únicas pistas a seguir, aunque éstas solo puedan aportar, desde un punto de vista metodológico, indicios para una cronología relativa cuyo espacio temporal no se puede concretar.

\section{UBICACIÓN}

La mina San Mateo, lugar conocido también como Son Creus, podría ser un punto notable de interés geológico y minero en Mallorca, sin embargo tras un siglo de abandono resulta un paraje de acceso extremadamente complicado. Además, la aproximación a los trabajos de 


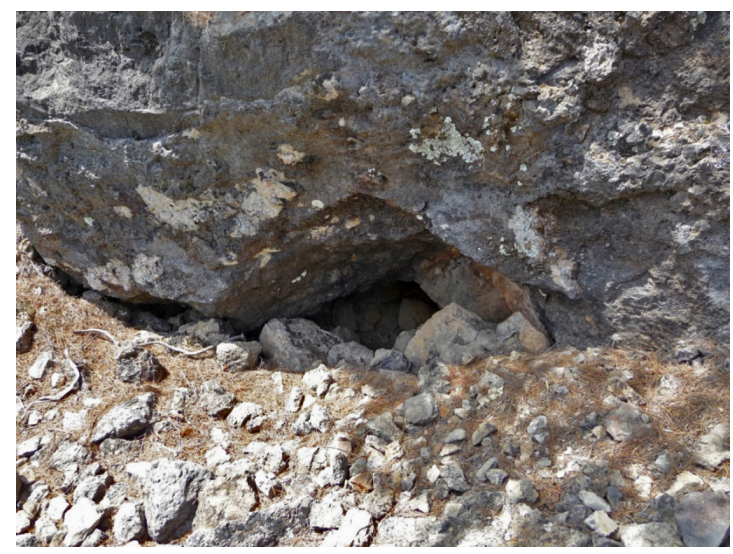

Fig. 2. Derrumbes recientes cegando una entrada o arranque de galería (Foto: M. H. Hermanns 2012).

interior entraña un riesgo físico nada desdeñable. Las últimas noticias escritas sobre la mina corresponden a 1992 (Merino 1992). Se realizó una intensa campaña de exploración espeleológica (fig. 1), en la cual el autor señaló la peligrosidad de la entrada a las zonas subterráneas. El estado actual es mucho peor del que existía entonces, con nuevos derrumbes que han cegado la práctica totalidad de las entradas (fig. 2).

El acceso a la zona minera se realiza desde la carretera Bunyola-Orient, aproximadamente a $1 \mathrm{~km}$ de aquella, tomando un camino que se desvía a la izquierda y a cincuenta metros, tras cruzar un arroyo por un pontón, desemboca en una zona de cultivo. Remontando cuarenta metros el arroyo, parte una pista hacia la izquierda ascendiendo hacia el paraje de Son Creus, un collado entre un cerro que domina Bunyola y la faja calcáreo-dolomítica donde se encuentra la mina, hacia el N.

Desde un gran claro, y remontando un sistema de estrechos bancales, se llega a la zona en cuestión. En estos bancales aparece material cerámico del que no hay referencias de haber sido datado. El hito más importante es una zona de escombros groseros de caliza en un claro rodeado de altos pinos (fig. 3). Es aquí la única zona, especialmente la parte inferior de la escombrera, donde han sido encontrados los pocos minerales de que disponemos: trozos de calamina del tamaño de un puño, nódulos de galena masiva y algunas impregnaciones de blenda. Remontando la escombrera por un angosto sendero, y superando un pequeño saliente rocoso, se accede al impresionante socavón de la mina (fig. 4). La parte oriental del socavón parece ser la más antigua, correspondiente al desmonte primitivo (¿romano?), mientras que la fachada
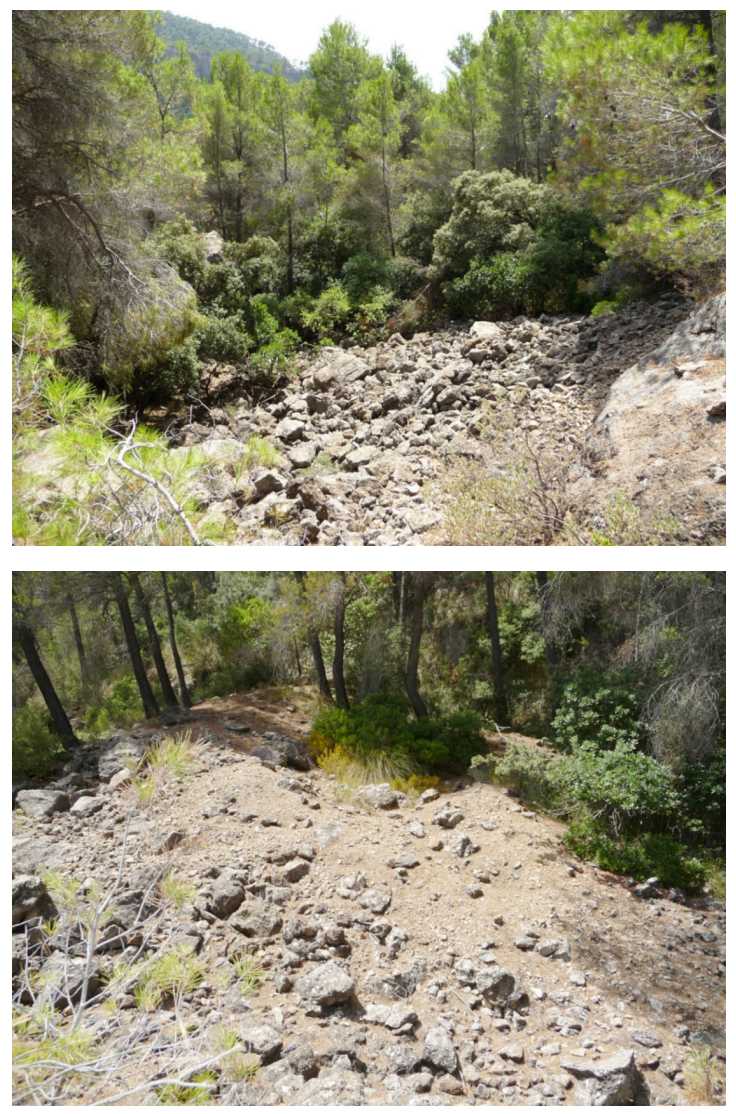

Fig. 3. Dos aspectos de la escombrera al pie del gran socavón en la región de la mina San Mateo, Son Creus, Bunyola (Palma de Mallorca) (Foto: M. H. Hermanns, 2012).

$\mathrm{N}$, un corte de más de $100 \mathrm{~m}$, es una sucesión de derrumbes naturales y deslizamientos en un sistema de estratos verticales, fallas y diaclasas subverticales. La sucesión de derrumbes han ido sepultando las numerosas entradas que existían; actualmente sólo quedan dos accesibles y una tercera sumamente peligrosa (en inminente colapso).

La parte más ancha del desmonte comprende una gran diaclasa de la que arranca hacia el fondo la que parece ser la entrada principal de la mina. La rampa está parcialmente rellena de escombros, pero al fondo parece haber una labor relativamente segura, realizada en roca calcárea, alternando con niveles menos competentes de margas compactas rojizas del triásico superior. Existe una segunda entrada en un extremo que parece relativamente segura y presenta huellas de rudimentarios barrenos (s. XIX) para el ensanche de la bocamina. En la parte $\mathrm{N}$ del desmonte aparecen varias bocaminas cegadas, así como 


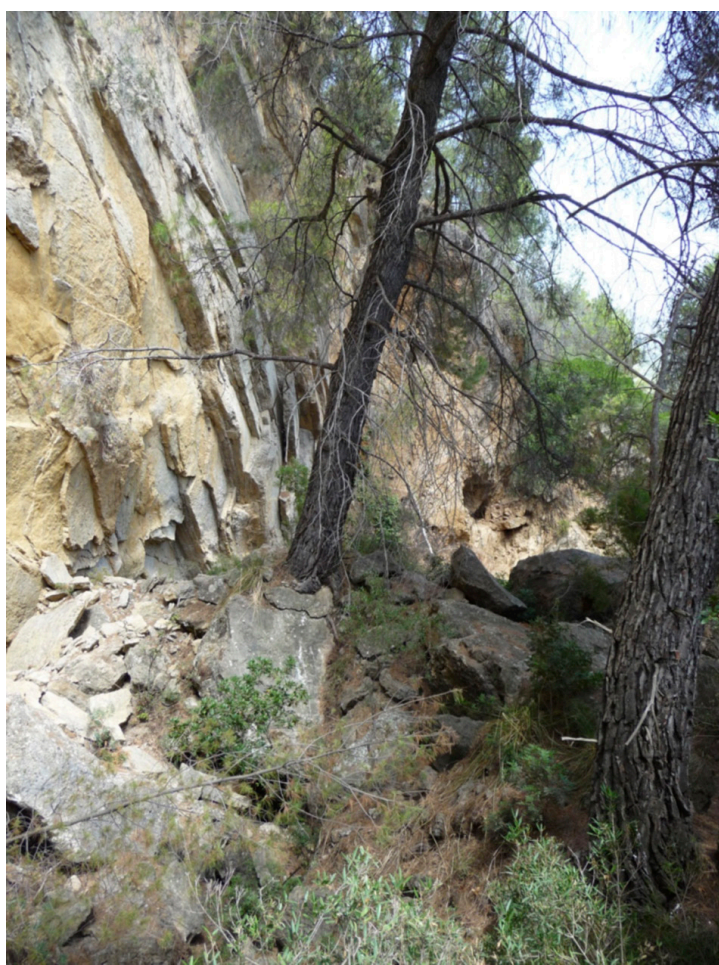

Fig. 4. Aspecto del gran socavón con derrumbes recientes (vista hacia el NE) (Foto: M. H. Hermanns, 2012).

una labor que ha vaciado una salbanda mineralizada vertical. Tiene más de diez metros de altura, y el fondo es una rampa muy vertical parcialmente tapada por grandes bloques.

De la infraestructura minera se conservan dos cabañas, parecidas a la documentada en el sector sur de la zona de s’Argentera, en Ibiza (fig. 5), cuya función debería ser la de refugio, diversas galerías (fig. 6) y la instalación de una posible trituradora (fig. 7).

\section{LA MINERÍA DE LOS SS. XIX Y XX}

En las décadas de 1840 y 1850 ya se conocen los principales indicios de minerales metálicos. $\mathrm{Al}$ parecer, en algunos puntos de la isla, existían riñones de piritas de hierro que presentarían huellas de haber sido trabajados, aunque no hemos vuelto a tener referencias a ellos. En los Anales de Minas de 1846 se lee:

Mina de galena llamada San Mateo. Se halla en el término de Buñols, con un pozo principal de 35 varas de profundidad y otro interior de nueve varas y dos cortas

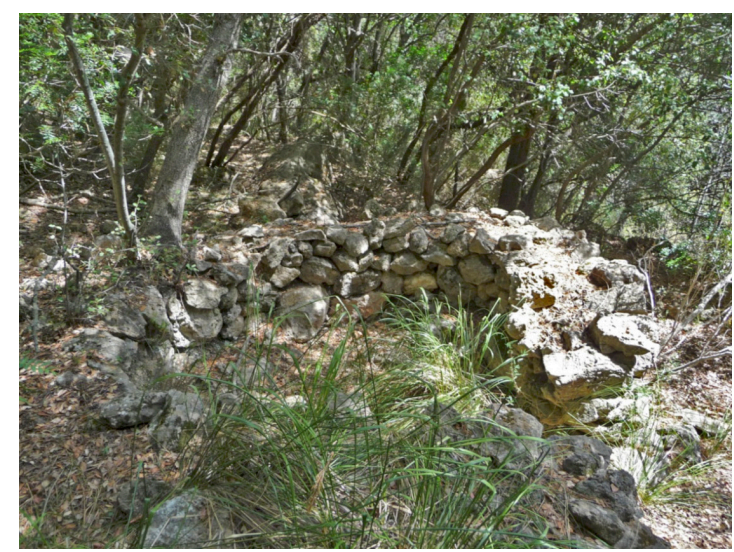

Fig. 5. La cabaña/el refugio (Foto: M. H. Hermanns, 2012).

galerías: explota un criadero de sulfuro de plomo, que aún no está suficientemente reconocido, haciendo poco tiempo que empezaron sus labores, las cuales continúan ocupándose en ellas 10 hombres.

Desde 1852 hasta 1863 no se tienen referencias a minas metalíferas en la actividad de la isla. Sin embargo, a partir de los años 60 del s. XIX hay información sobre la explotación de zinc (mina La Palmesana o Casualidad, Andratx), de cobre (mina de Lluch, Escorca) y de plomo (mina San Mateo) (para la documentación encontrada véase fig. 8). Además hay que mencionar la importancia del carbón mineral, cuyos yacimientos son conocidos en Mallorca desde 1811 gracias al informe del capitán de fragata Gerónimo Taverns; se explotaron para abastecer las grandes ciudades costeras de la península y para apoyar la industria de la navegación ante la creciente escasez de leña. Así, las primeras explotaciones del término de Benisalem se emprendieron en 1836 por cuenta de la Compañía Catalana de Navegación e Industria (Revista Minera 1863, 394).

La producción de mineral de plomo procedía de cuatro minas en la isla de Ibiza y de dos en la de Mallorca. Principalmente se trabaja en las tierras que rellenan antiguas labores (Estadística minera 1869: 42) y a medida que éstas van disminuyendo se abren nuevas zonas de trabajo en terrenos vírgenes (Estadística minera 1871: 39 con referencia a Ibiza). Sin embargo en Ibiza, y en concreto la Sociedad Esperanza, por carecer de planificación y haber realizado una explotación desordenada, ya en 1873 esta empresa se vio enfrentada a grandes dificultades para continuar explotando la obtención del mineral de manera rentable (Estadística minera 1873: 82). 

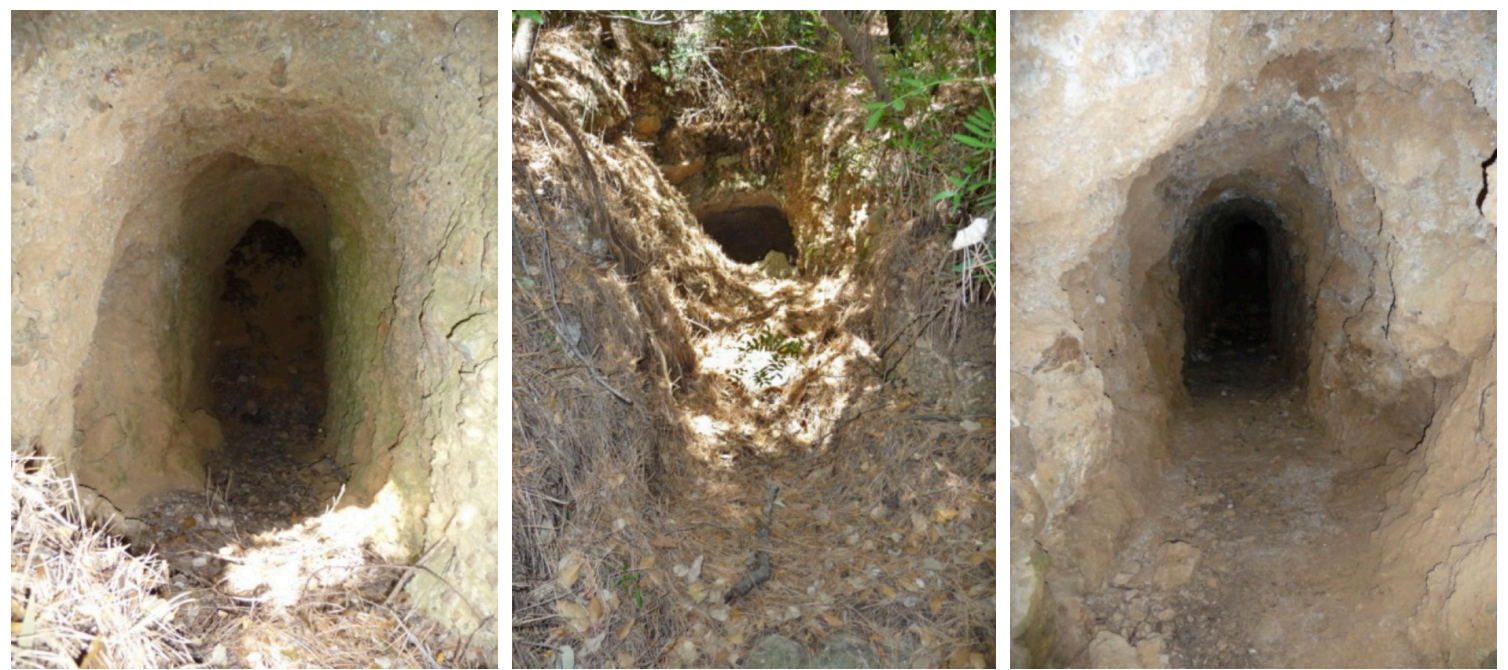

Fig. 6. Arranque de galerías de forma diversa, de momento sin datar (Foto: M. H. Hermanns, 2012).
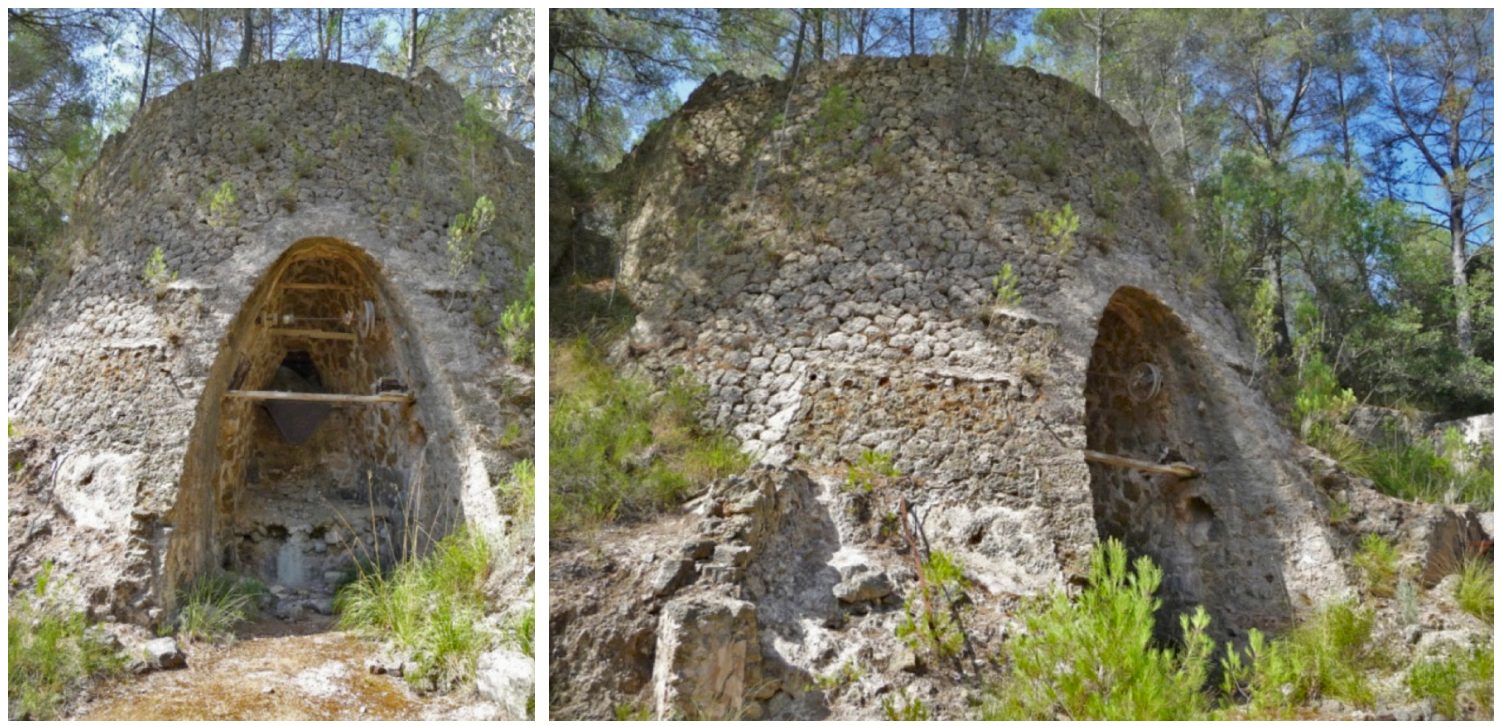

Fig. 7. Tolva de una posible trituradora ( $c f$. sin embargo el horno de yeso en Can Bleda, Sóller: Vicens et al. $2005: 66$ fig. 4 A) (Foto: M. H. Hermanns, 2012).

En 1874 una sociedad nominada La Conciliación Minera se hizo cargo de la concesión de la Minas San Mateo en Mallorca. Parece que el principal problema al que tuvieron que hacer frente los anteriores concesionarios fue el agua. Las labores antiguas se detenían exactamente en un nivel freático que estaba a $60 \mathrm{~m}$ de profundidad. La sociedad acomete la construcción de una galería de desagüe entre el arroyo cercano y la zona de trabajo. Esta sociedad vuelve a mencionar indicios de haberse hecho labores durante la dominación romana (Estadística Minera 1874: 73). A finales de ese año la galería tiene $400 \mathrm{~m}$ de desarrollo, pero aún no se había alcanzado la zona inferior de las labores antiguas. Según las exploraciones realizadas en ese momento, la mina antigua ocupaba una zona de $100 \mathrm{~m}$ de largo, $6 \mathrm{~m}$ de ancho y $60 \mathrm{~m}$ de profundidad. Al parecer el criadero mejoraba su ley a mayor profundidad. 


\begin{tabular}{|c|c|c|c|c|c|}
\hline carpeta & registro / expediente & lugar & nombre & mineral & año \\
\hline 7 & 0 & Bunyola & conciliación minera & plomo & 1889 \\
\hline 7 & -1 & Bunyola & santa rita, renuncia & hierro & 1889 \\
\hline 7 & 90 & Bunyola & santa rita & plomo & 1882 \\
\hline 7 & 112 & Bunyola & conciliación minera & plomo & 1873 \\
\hline 7 & 123 & Bunyola & conciliación minera & plomo & 1874 \\
\hline 7 & 290 & Bunyola & bunola & plomo & 1901 \\
\hline 7 & 412 & Bunyola & catalina & plomo & 1902 \\
\hline 7 & 467 & Bunyola & catalina & plomo & 1903 \\
\hline 7 & 508 & Bunyola & catalina & hierro & 1904 \\
\hline 7 & 526 & Bunyola & catalina & hierro & 1905 \\
\hline 7 & 568 & Bunyola & agregada & hierro & 1908 \\
\hline 7 & 752 & Bunyola & cordialidad & plomo & 1916 \\
\hline 7 & 760 & Bunyola & isabel & plomo & 1916 \\
\hline 7 & 1152 & Bunyola & san jose & indetermin & 1918 \\
\hline 7 & 1401 & Bunyola & san miguel & hierro & 1919 \\
\hline 7 & 1406 & Bunyola & remedios & hierro & 1919 \\
\hline 45 & 36 & Bunyola & consecuencia & plomo & 1876 \\
\hline 45 & 47 & Bunyola & virgen milagrosa & plomo & 1868 \\
\hline 45 & 49 & Bunyola & marcasita & hierro & 1877 \\
\hline 45 & 53 & Bunyola & productora & hierro & 1877 \\
\hline 45 & 66 & Bunyola & dichosa & hierro & 1878 \\
\hline 45 & 87 & Bunyola & motezuma & plomo & 1882 \\
\hline 45 & 88 & Bunyola & concepción & plomo & 1882 \\
\hline 45 & 93 & Bunyola & la mejoria & hierro & 1882 \\
\hline 45 & 98 & Bunyola & alfa & hierro & 1883 \\
\hline 45 & 110 & Bunyola & beta & hierro & 1883 \\
\hline 45 & 113 & Bunyola & la estrella & plomo & 1874 \\
\hline 45 & 115 & Bunyola & jupiter & plomo & 1874 \\
\hline 45 & 126 & Bunyola & consecuencia & hierro & 1885 \\
\hline 50 & 23 & Bunyola & virgen del amparo & zinc, calamina y plomo & 1867 \\
\hline 50 & 30 & Bunyola & san mateo & plomo & 1867 \\
\hline 50 & 31 & Bunyola & isabelita & plomo & 1867 \\
\hline 50 & 33 & Bunyola & san jose & plomo & 1868 \\
\hline 50 & 34 & Bunyola & carlota & hierro & 1876 \\
\hline 50 & 35 & Bunyola & lealtad & calamina & 1867 \\
\hline 50 & 37 & Bunyola & virgen de las nieves & calamina y plomo & 1867 \\
\hline 50 & $37,37 \mathrm{a}$ & Bunyola & simpatica & plomo & 1876 \\
\hline 50 & 536 & Bunyola & catalina & hierro & 1906 \\
\hline 50 & 1500 & Bunyola & catalina & hierro & 1925 \\
\hline 50 & 1505 & Bunyola & san miguel & plomo & 1926 \\
\hline 50 & 1506 & Bunyola & san miguel & hierro & 1926 \\
\hline
\end{tabular}

Fig. 8. Documentación sobre la minería de plomo en el Archivo del Distrito Minero de Baleares (Arxiu del Regne de Mallorca, Palma de Mallorca). 


\begin{tabular}{|c|c|c|c|c|c|c|c|c|c|}
\hline $\mathbf{N}^{0}$ laboratorio & $\mathrm{Na}_{2} \mathrm{O}$ & $\mathrm{BaO}$ & Sb & $\mathbf{P b}$ & MgO & $\mathbf{A l}_{2} \mathbf{O}_{3}$ & $\mathrm{SiO}_{2}$ & $\mathrm{P}_{2} \mathrm{O}_{5}$ & \\
\hline 5169_12 & 0,77 & 0,01 & 0,0004 & 83,2 & 1,35 & 0,03 & 0,29 & 0,003 & \\
\hline 5170_12 & 0,01 & $<0.003$ & 0,001 & 85,7 & 0,55 & 0,02 & $<0.11$ & 0,005 & \\
\hline 5171_12 & 0,008 & 0,003 & 0,0005 & 84,6 & 0,47 & 0,03 & $<0.11$ & 0,004 & \\
\hline 5172_12 & $<0.0015$ & 0,004 & 0,001 & 84 & 0,74 & 0,02 & $<0.11$ & 0,004 & \\
\hline 5173_12 & 0,004 & $<0.003$ & 0,001 & 82 & 2,29 & 0,02 & $<0.11$ & 0,003 & \\
\hline 5174_12 & $<0.0015$ & $<0.003$ & 0,0002 & 84,5 & 0,13 & 0,02 & $<0.11$ & 0,005 & \\
\hline $5175 \_12$ & 0,0017 & $<0.003$ & 0,0003 & 84,2 & 0,63 & 0,02 & $<0.11$ & 0,005 & \\
\hline 5176_12 & $<0.0015$ & $<0.003$ & 0,001 & 74,8 & 4,23 & 0,04 & 0,26 & 0,01 & \\
\hline $5177 \_12$ & $<0.0015$ & $<0.003$ & 0,0004 & 80,4 & 0,26 & 0,01 & 0,66 & 0,003 & \\
\hline $\mathbf{N}^{o}$ laboratorio & $\mathrm{Fe}_{2} \mathrm{O}_{3}$ & $\mathbf{S}$ & $\mathrm{CaO}$ & $\mathrm{TiO}_{2}$ & ZnO & $\mathrm{Cu}$ & $\mathrm{K}_{2} \mathrm{O}$ & As & Total en \% \\
\hline 5169_12 & 0,03 & 11,7 & 2,14 & 0,002 & 0,01 & $<0.002$ & 0,08 & 0,07 & 99,7 \\
\hline 5170_12 & 0,01 & 11,7 & 0,88 & 0,001 & 0,0004 & $<0.002$ & 0,03 & 0,001 & 98,8 \\
\hline 5171_12 & 0,01 & 11,8 & 0,75 & 0,001 & 0,01 & 0,002 & 0,02 & 0,001 & 97,7 \\
\hline 5172_12 & 0,07 & 10,4 & 1,24 & 0,0005 & 0,04 & $<0.002$ & 0,01 & 0,0004 & 96,5 \\
\hline 5173_12 & 0,03 & 11,1 & 3,56 & 0,001 & 0,03 & $<0.002$ & 0,01 & 0,001 & 99,1 \\
\hline 5174_12 & 0,01 & 12,5 & 0,19 & 0,0003 & 0,04 & $<0.002$ & 0,04 & 0,003 & 97,5 \\
\hline $5175 \_12$ & 0,01 & 11,7 & 1,07 & 0,0004 & 0,002 & $<0.002$ & 0,01 & 0,001 & 97,6 \\
\hline 5176_12 & 0,06 & 11,2 & 6,43 & 0,001 & 0,001 & $<0.002$ & 0,05 & 0,001 & 97,1 \\
\hline 5177_12 & 0,01 & 12 & 0,28 & 0,0005 & $<0.0003$ & $<0.002$ & 0,03 & 3 & 93,7 \\
\hline
\end{tabular}

Fig. 9A. Composición química en \% de las muestras tomadas.

\begin{tabular}{|c|c|c|c|c|c|c|c|c|c|c|}
\hline $\mathbf{N}^{0}$ laboratorio & $\mathrm{Sr}$ & $\mathbf{Y}$ & $\mathrm{Zr}$ & Nb & Mo & Ag & Cd & Sn & Sb & Te \\
\hline 5169_12 & 71 & 0,1 & $<1$ & 1 & $<0.5$ & 3,5 & 0,9 & 8 & 4,2 & 1,3 \\
\hline $\begin{array}{l}5170 \_12 \\
\end{array}$ & 58 & 0,1 & $<1$ & 0,7 & $<0.5$ & 1,3 & 1 & 13 & 9,5 & 0,5 \\
\hline 5171_12 & 29 & $<0.06$ & $<1$ & 0,6 & $<0.5$ & 1 & 0,7 & 7 & 4,9 & 0,4 \\
\hline 5172_12 & 145 & 0,1 & $<1$ & 0,5 & $<0.5$ & 3,9 & 0,7 & 5 & 6,2 & 1 \\
\hline 5173_12 & 81 & 0,1 & $<1$ & 0,5 & $<0.5$ & 0,8 & 0,6 & 4 & 6,3 & 0,2 \\
\hline 5174_12 & 15 & $<0.06$ & $<1$ & 0,5 & $<0.5$ & $<0.5$ & 0,7 & 8 & 2,4 & 0,7 \\
\hline 5175_12 & 120 & $<0.06$ & $<1$ & 0,7 & $<0.5$ & 1,8 & 0,7 & 10 & 2,9 & 0,4 \\
\hline 5176_12 & 125 & 0,2 & $<1$ & 0,5 & $<0.5$ & 1,2 & 1,1 & 5 & 14 & 0,7 \\
\hline 5177_12 & 18 & n.d. & n.d. & n.d. & n.d. & 0,3 & n.d. & 1,5 & 4 & 0,5 \\
\hline $\mathbf{N}^{\circ}$ laboratorio & $\mathbf{W}$ & $\mathbf{A u}$ & $\mathbf{B i}$ & $\mathbf{V}$ & $\mathrm{Cr}$ & Co & $\mathbf{N i}$ & Ga & Se & \\
\hline 5169_12 & 1,5 & 2,6 & 14 & 15 & 1,8 & 0,1 & 31 & 0,2 & $<4$ & \\
\hline 5170_12 & 0,9 & $<0.4$ & 14 & 1,1 & $<0.1$ & 0,02 & 29 & 0,3 & $<4$ & \\
\hline 5171_12 & 0,9 & $<0.4$ & 14 & 1,7 & $<0.1$ & 0,1 & 30 & 0,2 & $<4$ & \\
\hline 5172_12 & 0,7 & 1 & 14 & 17 & 1 & 0,1 & 58 & 0,3 & $<4$ & \\
\hline 5173_12 & 0,8 & $<0.4$ & 12 & 2,6 & $<0.1$ & $<0.04$ & 42 & 0,3 & $<4$ & \\
\hline 5174_12 & 0,6 & $<0.4$ & 14 & 0,3 & $<0.1$ & $<0.04$ & 61 & 0,2 & $<4$ & \\
\hline 5175_12 & 1,1 & $<0.4$ & 14 & 5,7 & $<0.1$ & $<0.04$ & 59 & 0,2 & $<4$ & \\
\hline 5176_12 & 0,7 & $<0.4$ & 11 & 3,3 & 0,3 & 0,03 & 71 & 0,3 & $<4$ & \\
\hline 5177_12 & n.d. & n.d. & 19 & n.d. & n.d. & 1 & 92 & n.d. & $<4$ & \\
\hline
\end{tabular}

Fig. 9B. Composición química en ppm de las muestras tomadas. 


\begin{tabular}{|c|c|c|c|c|c|c|}
\hline $\mathbf{N}^{\circ}$ laboratorio & ${ }^{206} \mathbf{P b} /{ }^{204} \mathbf{P b}$ & ${ }^{207} \mathbf{P b} /{ }^{204} \mathbf{P b}$ & ${ }^{208} \mathbf{P b} /{ }^{204} \mathbf{P b}$ & ${ }^{207} \mathbf{P b} /{ }^{206} \mathbf{P b}$ & ${ }^{208} \mathbf{P b} /{ }^{206} \mathbf{P b}$ & ${ }^{204} \mathbf{P b} /{ }^{206} \mathbf{P b}$ \\
\hline $5169-12$ & 18,71517 & 15,67345 & 38,7752 & 0,83734 & 2,071 & 0,05343 \\
\hline $5170-12$ & 18,71119 & 15,67769 & 38,77298 & 0,83765 & 2,07147 & 0,05344 \\
\hline $5171-12$ & 18,71999 & 15,67167 & 38,77325 & 0,83777 & 2,07183 & 0,05342 \\
\hline $5172-12$ & 18,71698 & 15,67333 & 38,77152 & 0,83784 & 2,07164 & 0,05343 \\
\hline $5173-12$ & 18,72246 & 15,67453 & 38,77273 & 0,83728 & 2,07159 & 0,05341 \\
\hline $5174-12$ & 18,72389 & 15,67622 & 38,76982 & 0,83711 & 2,07107 & 0,05341 \\
\hline $5175-12$ & 18,72478 & 15,67119 & 38,76885 & 0,83709 & 2,07109 & 0,05341 \\
\hline $5176-12$ & 18,72021 & 15,6737 & 38,76763 & 0,83748 & 2,07177 & 0,05342 \\
\hline $5177-12$ & 18,71259 & 15,67506 & 38,76749 & 0,83752 & 2,07143 & 0,05344 \\
\hline
\end{tabular}

Fig. 10. Proporciones de isótopos de las muestras tomadas.

Entre 1875 y 1876 continúan las labores en dos frentes, por un lado trabajando en la zona ya explotada, y por otro en la profundización de la galería, para lo que se destina a cuatro hombres. En el año 1878 se produce una crisis en el precio del plomo, lo que casi lleva al cierre de las minas de Baleares (Estadística minera 1878: 71). En Bunyola se suspendieron los trabajos en la galería porque las condiciones de trabajo comenzaban a resultar penosas debido a la mala ventilación. Sólo había una entrada y no habían logrado enlazar con los trabajos antiguos. Una explosión de gas en la galería llevó finalmente a la decisión de detener la explotación. Ya no volverá a nombrarse en la documentación una posible explotación del mineral. Sin embargo, por ser el criadero de metales más importante de Mallorca, será motivo de estudios e investigaciones posteriores, aunque nunca superará la fase de permiso de investigación.

En 1906 se analizan nuevamente sus calaminas y en 1907, aprovechando la reactivación de las minas de Ibiza, se extrajeron algunas toneladas de mineral de plomo de las escombreras antiguas de Bunyola (Estadística minera 1907: 108). Entonces se lleva a cabo una nueva campaña para habilitar algunas labores y terminar la galería. Sin embargo el yacimiento no tuvo continuidad, limitándose a explotar la zona que ya había sido explorada. Se abandona definitivamente esta mina que tanto interés había despertado a lo largo de los siglos. Mientras que en 1945 se reanudan (puntualmente) las investigaciones en Ibiza, parece que en Bunyola no fue así (Estadística Minera 1945: 237).

Mientras que el criadero de Ibiza ya se menciona en 1791 en los Anales del Real Laboratorio de Química de Segovia, escrito por Louis Proust, a raíz de unas muestras tomadas para el Ensayo sobre algunas minas de plomo de
España (Proust 1791: 38 s.), no parece que éste fuera el caso de las minas de Bunyola. Un siglo más tarde, el Archiduque Luis Salvador en su obra Die Balearen geschildert in Wort und Bild (von Habsburg 1897: 339 s.), menciona Bunyola con una sola mina de plomo en servicio (La Virgen de Amparo) de tres que había en toda la isla. La minería de la isla de Mallorca estaba claramente enfocada a la explotación de las salinas y del lignito, producciones mucho más rentables y fructíferas, como queda patente en la Estadítica Minera de los años en cuestión.

\section{LA CONTEXTUALIZACIÓN ARQUEOLÓGICA}

Para determinar la signatura isotópica del criadero, se tomaron y analizaron un total de nueve muestras de galena procedentes de la escombrera de la mina con material estéril localizado al pie del socavón grande. Se determinaron su composición química (fig. 9a y b), y las proporciones de isótopos de plomo (fig. 10) mediante LIA (MC-ICP-MS, Neptuno, Thermo, en los laboratorios del Deutsches Bergbaumuseum (Museo Alemán de Minería), en Bochum, y los de la Universidad de Frankfurt am Main.

Sabiendo que la explotación de galena en la Antigüedad en gran parte fue para la obtención de plata, llama la atención la bajísima cantidad de plata de las muestras localizadas en la escombrera de Bunyola. En la Figura 11 se reflejan las proporciones de los isótopos. A modo de comparación, los datos se contrastaron con los campos isotópicos de otras cuencas mineras de la península Ibérica. Los minerales procedentes de Bunyola forman un grupo muy homogéneo. En el gráfico se percibe que la zona de Bunyola 
Fig. 11. Localización de la huella isotópica del criadero de Bunyola (Mallorca) en comparación con las conocidas de la península Ibérica (gráfico: I. Montero Ruíz, CSIC Madrid).

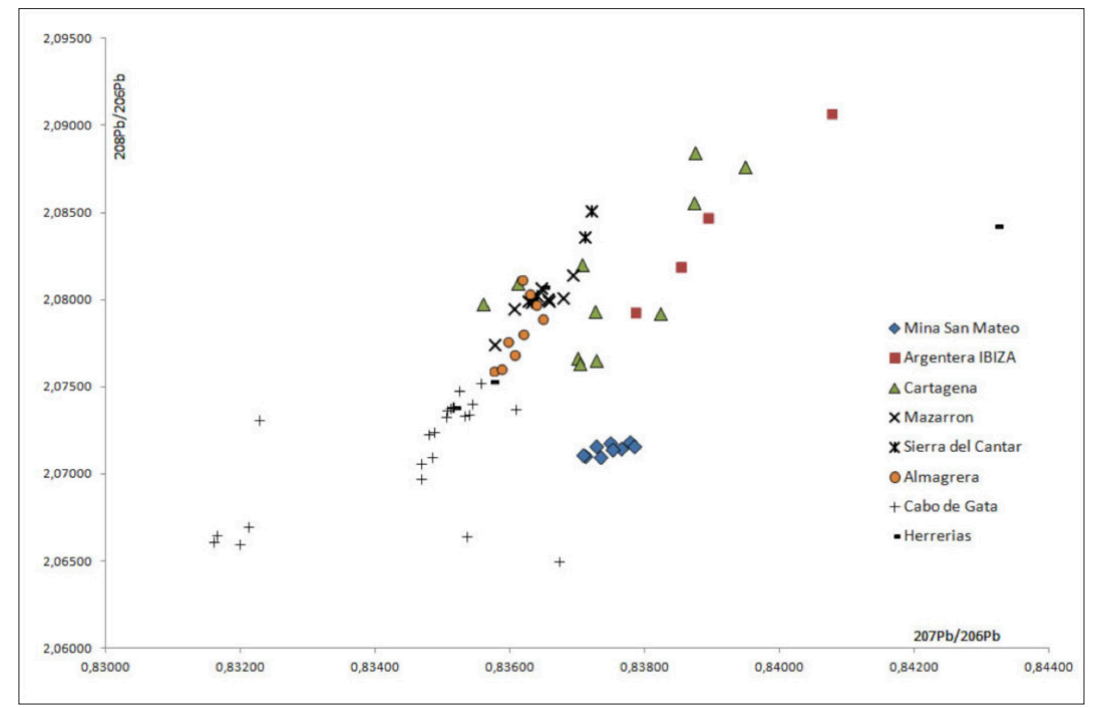

hasta el momento se diferencia fácilmente del resto de las minas peninsulares, especialmente del noreste de la isla de Eivissa (criadero de s'Argentera).

Para establecer una comparación con materiales arqueológicos se seleccionaron cuatro lingotes de plomo, de los cuales se discutió una posible procedencia balear. Se trata de tres lingotes de plomo de posible datación en época tardorromana, procedentes de una localidad cercana a Bunyola, y un lingote de plomo de época romana procedente del naufragio Escombreras III, hallado en la rada del puerto de Cartagena ${ }^{1}$ (fig. 12).

Los tres lingotes mallorquines son de forma rectangular (Museo de Mallorca, Inv. 15355, Proyecto E-150; medidas: 36 × 23,5 x 3,5 cm, peso: 17,6 kg) o elíptica (Museo de Mallorca, Inv. 15356, Proyecto E-151; medidas: 38,5 x 30 x $3 \mathrm{~cm}$, peso: 24,4 kg y Museo de Mallorca, Inv. 15357, Proyecto E-152; medidas: 40 x 24 x 3,5 cm, peso: 21,1 $\mathrm{kg}$ ), todos con perfil planoconvexo procedentes de Ses Fontenelles (Santa María del Camí) hallados alrededor de 1935 durante unas labores agrícolas. El contexto es incierto, aunque de la zona se conocen diversas estructuras de índole arqueológica, así como hallazgos en superficie que inducen a pensar en un asentamiento rural con necrópolis. Los tres lingotes presentan una inscripción en hebreo Šemuel, Hijo de R. Haggai y unas marcas X y X con círculo (Millás Vallicrosa 1958: 3-9; Veny 1965: 23-24 Cat. nº 12 a, b, c; Planas-Forcano 2009; Noy 1993: 238-239 nº 177, sheet 1, 2, 3). Posiblemente los lingotes provengan de enterramientos (Noy 1993: 238, epitaph on lead sheets - Zucca 1998: 202 - Orfila 2006: fig. en p. 222: lauda sepulcral) de un posible asentamiento judío. José María Millás Vallicrosa (1958) propone una datación en el s. IV ó V d.C. por razones paleográficas, mientras que David Noy (1993) no descarta una datación anterior (sobre la comunidad judía de Mallorca en época tardorromana, $c f$. Panzram 2013, 27). Debido a su proximidad a la mina de Bunyola, que se encuentra al O del lugar del hallazgo, David Noy propuso además una producción local de estos lingotes.

El pecio de Escombreras III se fecha en la primera mitad del s. I a.C. En él se descubrieron cuatro lingotes de plomo, dos de ellos mencionan a C. Aquinius M.f. y otros dos a L. Planius L. F. Ruscinus y la SOC(ietas) BALIAR(icae?) (Díaz 2008: 276). Más allá de lo propuesto por Antonio M. Poveda, quien propuso ver aquí una sociedad de publicani (arrenderos de derechos mineros), que comerciaban en las islas Baleares (Poveda 2000). Sin embargo, también se podría pensar en una sociedad que explotara los recursos mineros en las propias Baleares, siendo la inscripción central en los lingotes de esta forma la del productor, según los estudios de Claude Domergue (veáse Rico 2011). Visto que es a partir de la mitad del s. I d.C. cuando se impuso la distinción entre insulae baliarides y gymnasiae insulae (Estrabón III 5.1; cf. Zucca 1998: 16-23) cabría pensar en un origen tanto pitiuso como balear propiamente dicho. Sin embargo, no hay una interpretación satisfactoria para esta inscripción, porque no puede descartarse su conexión con los precintos de plomo encontrados en las minas de Castuela (Badajoz) y Santa Bárbara (Córdoba) en los que también aparecen las siglas S. BA. (Díaz 2008: 291). 


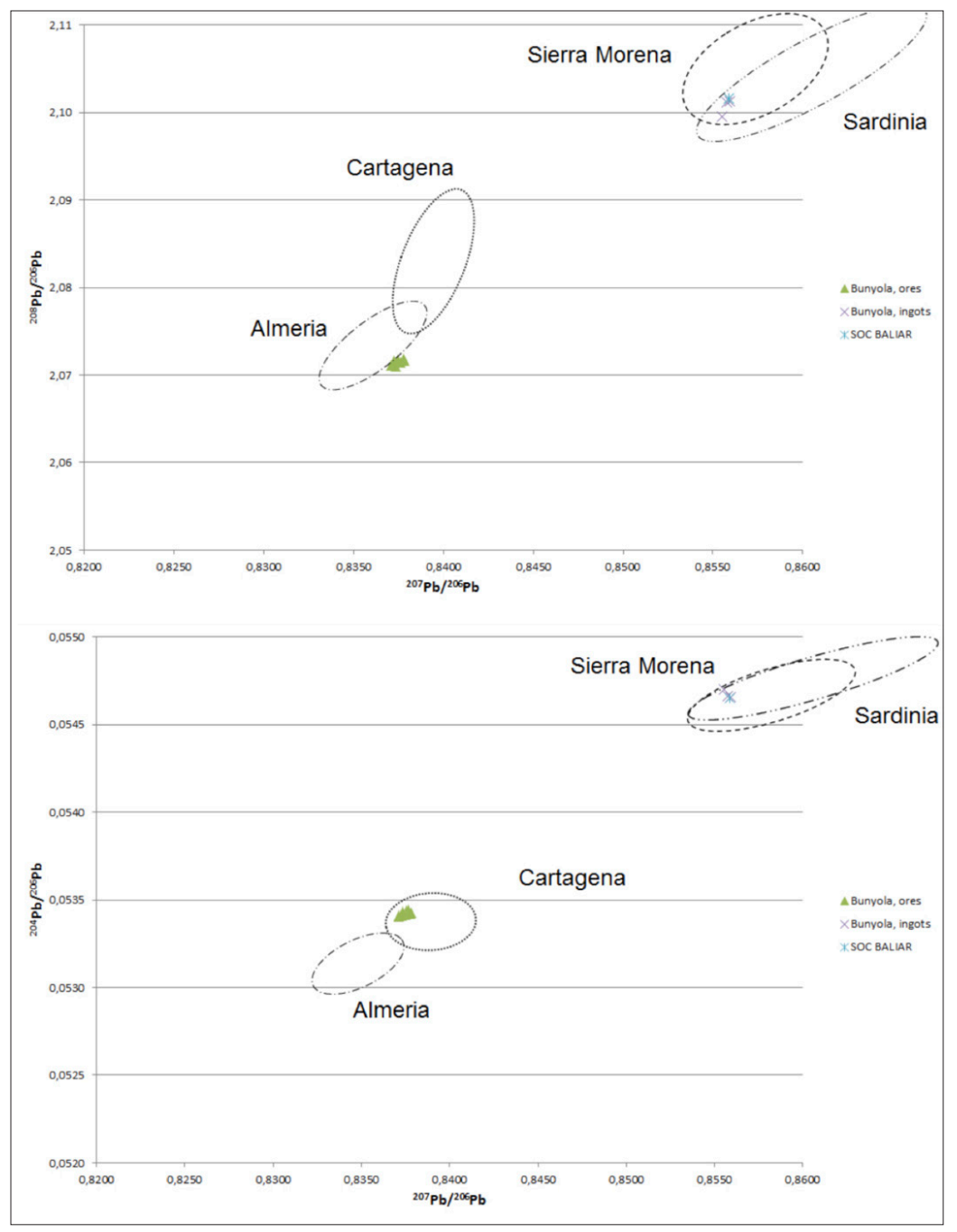

Fig. 12. Localización isotópica de los lingotes de plomo de Bunyola y el del pecio de Escombreras (Gráfico: M Bode, DBM, Bochum). El margen de error o desviación estándard se ubica dentro del tamaño del símbolo.

El gráfico de la Figura 12 refleja que una procedencia de los lingotes, tanto los de Bunyola como el del pecio de Escombreras III, que en un principio se podían relacionar con los yacimientos mineros de las islas Baleares por la localización topográfica de los hallazgos o por su lectura epigráfica, de momento queda descartada. En la figura puede verse que los lingotes hallados en Bunyola correlacionan bien con los lingotes del pecio romano correspondiendo a una mineralización de mayor edad geológica tal como se conocen de la zona de Sierra Morena o Cerdeña. Sin embargo, también se refleja que éstos no se corresponden con la mineralización obtenida para Bunyola y por lo tanto habría que descartar de momento una elaboración de minerales procedentes del yacimiento mallorquín.
La mención de labores romanas en la bibliografía minera hizo que el criadero de Bunyola apareciera mencionado en la bibliografía arqueológica sin que se hubieran localizado concretamente las labores antiguas sobre el terreno (Domergue 1990: 187, 46 s. fig. 40 PM 1; Domergue 1990: 203; Meier 1995: 63 s.) ni que hubiera contextos arqueológicos con objetos atribuibles a éste. Es la razón por la cual se procedió a la búsqueda de análisis de objetos arqueológicos de plomo para confrontarlos con los análisis de mineralizaciones del propio criadero. Ahora cabría aumentar el muestreo en un futuro próximo con más material de posible fabricación local, como podrían ser los objetos de plomo de época prehistórica o medieval. En la Antigüedad, con el plomo se producían objetos muy diversos: caben 
mencionar las plaquetas de plomo de época prehistórica (Enseñat 1975), y los glandes de plomo del conocido hondero balear (Quesada 1997: 475-480, especialmente p. 478 s.; Planas 1994). Sin embargo hay que tener en cuenta el problema metodológico que presentan los glandes de plomo, ya que al ser objetos de fácil fabricación en el campo, constatada arqueológicamente, hay muchas posibilidades de que se hayan realizado con metal reciclado de muy diversa procedencia. De época bizantina cabe mencionar las bulas de plomo halladas en el castillo de Santueri (Nadal 2006). También mencionar que durante el Alto Medievo el plomo fuera un componente del barniz utilizado en la cerámica esmaltada mallorquina.

\section{CONCLUSIONES}

El elemento más importante de los avances aquí presentados es, sin duda, el estudio arqueométrico y la contribución a la caracterización de la signatura isotópica de las minas de Bunyola. La identificación de yacimientos mineros menores, como el de Bunyola, y la caracterización de otros yacimientos de plomo de las Baleares (Ibiza) y de la península Ibérica (Cartagena, Almería, Sierra Morena, Priorat) a partir de la analítica es algo esencial para futuros estudios, ya que contribuyen a una visión general del paisaje minero. Por ello debería haber más referencias para encuadrar mejor los resultados obtenidos con los análisis de piezas documentadas arqueológicamente. La constatación analítica de que los lingotes de Bunyola y el del pecio romano de Escombreras III no coinciden con el campo isotópico de Bunyola, es la razón por la cual de momento no se puede contrastar la explotación de la mina en época romana.

En la Estadística Minera del año 1931 se lee: En la isla de Mallorca y en la de Ibiza existen criaderos (de plomo) conocidos desde la época fenicia y trabajados posteriormente por los romanos, como atestiguan numerosas monedas, candiles y otros objetos de esa época encontrados en las excavaciones. Referente a Bunyola, se hace hincapié en el hecho de que al haber considerables escombreras, las labores en época antigua tuvieron que ser my intensas (Estadística Minera 1931: 92-93).

Una vez más la discrepancia entre la no coincidencia puntual de los resultados analíticos con los relatos mineros nos hacen ser prudentes a la hora de la interpretación histórica. Como en otras partes de la península Ibérica, y no solo en las Baleares, se hace mención a menudo de labores anteriores. Sin embargo, hay que tener en cuenta que en la época del auge minero fue un procedimiento común denominar cualquier explotación como romana, lo que parece que le daba solera a la mina en cuestión (cf. Jáuregui 1948: 89). En algunas ocasiones, las cerámicas (lucernas romanas y bizantinas, fragmentos de ánforas vinarias de factorías púnicas ibicencas) y otros restos arqueológicos hallados en las galerías permiten deducir que estas explotaciones antiguas fueron más intensivas que las del Medievo (Encinas 2007: 202).

Por último señalar que, debido al reducido número de muestras, sólo se pueden sugerir las posibles relaciones y procedencias, pero sin pretender una identificación segura. Los datos necesarios para ello deberán proporcionarlos otros análisis complementarios que se realicen sobre una base más segura.

\author{
Marcus Heinrich Hermanns \\ Instituto Arqueológico Alemán \\ (Deutsches Archäologisches Institut) \\ Marcus.Hermanns@dainst.de
}

\section{NOTA}

1. Se trata de tres lingotes analizados dentro del Proyecto Corpus Massarum Plumbearum Romanarum (Corpus de los lingotes de plomo romanos) de la Kommission für Alte Geschichte und Epigraphik des Deutschen Archäologischen Instituts y del Deutsches Bergbau-Museum de Bochum, cuyos datos me fueron facilitados amablemente por Peter Rothenhöfer (DAI; AEK München) y Michael Bode (DBM).

\section{AGRADECIMIENTOS}

Este trabajo ha sido posible gracias a las aportaciones de diversas personas e instituciones, a las cuales estoy sinceramente agradecido: al Instituto Arqueológico Alemán por su apoyo financiero y logístico para llevar a cabo estos trabajos; a Ignacio Montero (CSIC, Madrid), por la información y el gráfico; a Luis Jordá Bordehore por sus indicaciones, a tavés de diferentes entrevistas, sobre la minería mallorquina.

\section{BIBLIOGRAFÍA}

DÍAZ, B. (2008): Epigrafía latina republicana de Hispania, Instrumenta 26, Barcelona.

DOMERGUE, C. (1987): Catalogue des mines et des fonderies antiques de la Péninsule Iberique I, Publications CDV, Madrid. 
DOMERGUE, C. (1990): Les mines de la péninsule Ibérique dans l'antiquité romaine, CEFR 127, Roma.

ENCINAS, J. A. (2007): La incidencia antrópica en las cavernas baleáricas, Mallorca.

ENSEÑAT, C. (1975): Las plaquetas de plomo mallorquinas, sistematización tipológica, Majurqa 1975, 63-117.

ESTADÍSTICA MINERA de España (ed. Instituto Geológico de España, Madrid)

von HABSBURG, L. S. (1897): Die Balearen geschildert in Wort und Bild, Leipzig/Würzburg.

HERMANNS, M. H. (2013): Forschungsperspektiven der Montanarchäologie auf den Balearen: Antike Blei- und Silbergewinnung auf Ibiza, Madrider Mitteilungen 54, 243-275.

JÁUREGUI, J. J. DE (1948): Minería antigua en Cabo de Palos, Congreso Arqueológico del Sudeste Español, Murcia 1947, Cartagena, 79-97.

JORDÀ, L. (2003): Notas históricas para el estudio de la minería metálica en Mallorca (1846-1909), Libro de Actas 1er. Simposio latino sobre Minería, Metalurgía y Patrimonio Minero en el Mediterráneo Occidental (SEDPGYM, ed.), La Pobla del Segur, 119-130.

JORDÀ, L.; HERMANNS, M. H.; JORDÀ, R. (2011): Apuntes para el conocimiento histórico de las minas de plomo argentífero de s'Argentera (Ibiza) en los siglos XIX y XX, De Re Metallica 17, 1-11.

MEIER, St. W. (1995): Blei in der Antike. Bergbau, Verhüttung, Fernhandel, Tesis doctoral, Universidad de Zürich.

MERINO, A. (1992): Aportación al conocimiento de la mina "San Mateo" (Bunyola, Mallorca), Endins 17-18, 89-95.

MILLÁS, J. (1958): Los plomos con inscripción hebraica de Ses Fonteneles (Mallorca), Sefarad 18, 3-9.

NADAL, J. (2006): Las bulas de plomo bizantinas del Castillo de Santueri, Bolletí de la Societat Arqueològica Lul-liana 62, 325-340
NOY, D. (1993): Jewish inscriptions of western Europe I. Italy, Spain and Gaul, Cambridge.

ORFILA, M. (2006): Las Baleares durante el Bajo Imperio y la Antigüedad Tardía, Historia de las Islas Baleares IV. Las Baleares en época romana y tardoantigua, Palma de Mallorca, 209-249.

PANZRAM, S. (2013): Kleine Geschichte der Balearen, Klio 95.1, 5-39.

PLANAS, A. (1994): La útil honda balear nutrida de plomo (Silio Italico), Eivissa.

PLANAS, S.; FORCANO, M. (2009): A history of Jewish Catalonia: the life and death of Jewish communities in Medieval Catalonia, Girona.

POVEDA, A. M. (2000): Societas Baliarica. Una nueva compañía minera romana de Hispania, Gerión 18, 293-313.

PROUST, L. (1791): Anales del Real Laboratorio de Química de Segovia, ó Colección de memorias sobre las artes, la artillería, la historia natural de Espańa, y Américas, la docimástica de sus minas, etc. I, Segovia.

RAMÓN, J.; RAFEL; N.; MONTERO, I.; SANTOS, M.; RENZI, M.; HUNT, M.A.; ARMADA, X. L. (2011): Comercio protohistórico: el registro del Nordeste Peninsular y la circulación de mineral de plomo en Ibiza y el Bajo Priorato (Tarragona), SAGVNTVM-PLAV 43, 55-81 DOI: http://dx.doi.org/10.7203/SAGVNTVM.43.1644

QUESADA, F. (1997): El armamento ibérico. Estudio tipológico, geográfico, functional, social y simbólico de las armas en la cultura Ibérica (Siglos VI-I a.C.), Montagnac.

VENY MELIA, C. (1965): Corpus de las inscripciones baleáricas hasta la dominación árabe, Roma.

VICENS, D.; CRESPÍ, D.; BOVER, P.; GINARD, A.; VADELL, M.; BARCELÒ, M. A. (2005): Les Cavitats de la Serra de Na Burguesa. Zona 7: Les Coves del Pilar i les mines de guix (Palma, Mallorca), Endins 27, 47-74.

ZUCCA, R. (1998): Insulae Baliares: le isole Baleari sotto il dominio romano, Roma. 